\title{
Los peces Clupeiformes del litoral valdiviano (Chile): clave de reconocimiento y comentarios (Pisces: Osteichthyes)
}

\author{
Clupeiform fishes of the Valdivian littoral (Chile): Key for identification and comments \\ (Pisces: Osteichthyes)
}

\author{
Sergio Silva G. ${ }^{1}$ y Germán Pequeño R. ${ }^{2}$ \\ ${ }^{1}$ Departamento de Acuicultura, Subsecretaría de Pesca, Bellavista 168, piso 17, Valparaíso, Chile \\ ${ }^{2}$ Instituto de Zoología “Ernst F. Kilian”, Universidad Austral de Chile, Casilla 567, Valdivia, Chile \\ gpequeno@uach.cl
}

\begin{abstract}
Two families and four species of clupeiform fishes are recognized for the Valdivian coast (Chile), on the basis of the revision of ichthyological collections. These are: Family Engraulidae, with Engraulis ringens and Family Clupeidae with Ethmidium maculatum, Sardinops sagax and Strangomera bentincki. A series of characteristics selected by its stability are presented as the most advisable, for the
\end{abstract}

taxonomical identification work. An illustrated taxonomic key is shown, as well as details about the morphological and meristic characteristics.

Key words: Sardines, anchovies, Clupeiformes, Valdivia, Chile

\section{Introducción}

Los peces del orden Clupeiformes, que habitualmente incluyen a las sardinas, machuelos o tritres, anchoas o anchovetas, etc., constituyen uno de los grupos de peces de mayor importancia en la pesca mundial y local. Desde antaño, dichos peces han sido capturados, principalmente con fines de alimentación y obtención de una serie de subproductos de interés comercial (Whitehead et al., 1988). En el Océano Pacífico sur oriental, principalmente frente a Chile y Perú, han pasado a constituir registros históricos de capturas, probablemente las de mayor magnitud en la historia (Simpson et al. 1967, Whitehead 1985, Yánez et al. 1997).

En los primeros decenios (1950-1970) en que dichas capturas empezaron a incrementarse a raíz del aumento de la producción de harina de pescado, las pesquerías más importantes de clupeiformes se concentraron en la zona norte de Chile (Mann 1954). Pero, a medida que las poblaciones (= stocks) empezaron a mostrar declinaciones en su abundancia para la pesquería, la actividad extractora se fue trasladando poco a poco hacia el sur del país, hacia zonas donde incluso el público común pensaba que este tipo de peces no existía, lo cual han observado directamente los autores al ver los puertos de origen de las embarcaciones trabajando en la costa de Valdivia, en los últimos diez años.

En los últimos dos decenios del siglo $\mathrm{XX}$ y principios del presente, no solamente se ha constatado que en la zona al sur del golfo de Arauco existen clupeiformes, sino se ha llegado a establecer que hay mas de dos especies (Ethmidium maculatum, Sprattus fuegensis y Strangomera bentincki) (Pequeño \& Sáez, 2004). Sin embargo, para diversos fines, resulta difícil reconocer estos peces en terreno, pues las especies involucradas son bastante parecidas entre sí y existen pocas herramientas para poder hacer la diferenciación en el mismo lugar de trabajo y, aún resulta complicado reconocer ejemplares en el laboratorio, especialmente cuando éstos han sido deformados por causas de la misma manipulación de captura. Autores como De Buen (1958), Leible \& Alveal (1982), Whitehead et al. (1988) y otros, han hecho claves y descripciones sobre la base de proporciones morfométricas y elementos merísticos de fácil cuantificación. Sin embargo, el hecho que en dichas descripciones no se considere la variabilidad intraespecífica de algunos elementos de utilidad en las diferenciaciones taxonómicas (por ej. longitud de la boca), es un problema que se acentúa con la falta de un número significativo de ejemplares. 
Por lo anterior, nuestro objetivo es señalar con documentación biológica, cuáles son las especies que habitan en aguas valdivianas, acompañando una clave de determinación taxonómica de aquellas especies y, discutir brevemente, aspectos relevantes de este grupo en la región.

\section{Material y métodos}

Se considera como litoral valdiviano, a aquel que se extiende aproximadamente entre Isla Mocha (38 $22^{\prime}$ 'S, $\left.73^{\circ} 43^{\prime} \mathrm{W}\right)$ y Carelmapu ( $41^{\circ} 45^{\prime} \mathrm{S}$, 38 $22^{\circ} \mathrm{W}$ ), en una aproximación a lo que corresponde al sector sur del distrito biogeográfico centro-chileno (López 1963, Pequeño 2001, 2004). Se revisaron 170 ejemplares de Clupeiformes capturados en la zona de Valdivia, existentes en la Colección de Peces Marinos del Instituto de Zoología "Ernst F. Kilian” de la Universidad Austral de Chile. Se obtuvieron datos sobre números de catálogo, año, lugar de captura y número de ejemplares de cada muestra (Tabla 1).

Tabla 1

Ejemplares de clupeiformes del litoral valdiviano analizados en éste estudio, con indicación del número de catálogo (IZUA-PM), año de captura, lugar y número de ejemplares

Specimens of clupeiform fishes from the Valdivian littoral analyzed in this study, with indication of catalog number (IZUA-PM), year of capture, place and number of specimens

\begin{tabular}{|c|c|c|c|c|}
\hline Especies & IZUA-PM & Año & Lugar & $\mathrm{n}$ \\
\hline \multirow[t]{9}{*}{ Engraulis ringens } & 999 & 1975 & Corral & 2 \\
\hline & 649 & 1978 & Mehuín & 2 \\
\hline & 671 & 1979 & Mehuín & 3 \\
\hline & 1295 & 1984 & Corral & 8 \\
\hline & 1296 & 1984 & Calbuco & 1 \\
\hline & 1200 & 1984 & Calbuco & 13 \\
\hline & 1202 & 1984 & Mehuín & 4 \\
\hline & 935 & 1985 & Mehuín & 1 \\
\hline & 1218 & 1989 & Corral & $\begin{array}{c}16 \\
n=50\end{array}$ \\
\hline \multirow[t]{11}{*}{ Ethmidium maculatum } & 389 & 1975 & Mehuín & 1 \\
\hline & 528 & 1977 & Mehuín & 7 \\
\hline & 552 & 1977 & Corral & 2 \\
\hline & 574 & 1978 & Mehuín & 1 \\
\hline & 787 & 1979 & Corral & 13 \\
\hline & 845 & 1984 & Mehuín & 5 \\
\hline & 867 & 1984 & Mehuín & 1 \\
\hline & 1362 & 1985 & Corral & 13 \\
\hline & 990 & 1987 & Corral & 5 \\
\hline & 1223 & 1989 & Mehuín & 2 \\
\hline & & & & $\mathrm{n}=50$ \\
\hline \multirow[t]{8}{*}{ Strangomera bentincki } & 6 & 1960 & Corral & 1 \\
\hline & 89 & 1960 & Corral & 22 \\
\hline & 78 & 1962 & Corral & 1 \\
\hline & 79 & 1962 & Corral & 4 \\
\hline & 949 & 1984 & Corral & 8 \\
\hline & 1038 & 1984 & Corral & 6 \\
\hline & 1229 & 1989 & Corral & 8 \\
\hline & & & & $\mathrm{n}=50$ \\
\hline \multirow[t]{4}{*}{ Sardinops sagax } & 795 & 1979 & Corral & 5 \\
\hline & 1037 & 1984 & Corral & 14 \\
\hline & 1363 & 1991 & Curiñanco & 1 \\
\hline & & & & $\mathrm{n}=20$ \\
\hline
\end{tabular}


El reconocimiento taxonómico de cada ejemplar, se hizo siguiendo los trabajos de De Buen (1958), Boré \& Martínez (1980), Leible \& Alveal (1982), Chirichigno \& Vélez (1998), Whitehead (1964, 1985), Sáez (1988) y Whitehead et al. (1988). Las medidas morfométricas y merísticas se seleccionaron a través del coeficiente de variación (CV) (Sokal \& Rohlf 1979):

$$
\mathrm{CV}=\frac{\text { DE de “y” x } 100}{\text { Promedio de la variable “y” }}
$$

donde

DE = desviación estándar

$\mathrm{y}$ = media aritmética de una variable dada

Con el fin de determinar el ajuste de las distribuciones de frecuencias a la curva de normalidad, se siguió un método convencional propuesto por Harding (1949), aplicado y modificado por Figueroa (1985). Este método gráfico requiere la estimación de la probabilidad del rango medio (PRM), que se calculó según la fórmula siguiente:

$$
\mathrm{PRM}=\frac{\text { Frecuencia acumulada }}{\mathrm{N}+1} \times 100
$$

donde

$\mathrm{N}=\mathrm{N}^{\mathrm{o}}$ de ejemplares

Aquellas características que presentaron alta estabilidad (CV inferior al 10\%), en las especies analizadas, fueron seleccionadas como útiles para el trabajo comparativo. Según las necesidades, se usaron otros estadísticos, como promedio, rango y ecuación de regresión simple.

Luego del reconocimiento taxonómico de los ejemplares, se hizo una selección de las características morfológicas con carácter diferencial y con capacidad para discriminar de manera directa, especialmente por observación a ojo desnudo. Hecha la mencionada selección, se preparó una clave dicotómica para reconocer las especies de interés, preparándose esquemas con el fin de darle mayor funcionalidad y posibilidades de uso. En las distintas especies encontradas, también se entrega un resumen de características diferenciales fundamentales, de fácil acceso.

Tabla 2

Medidas y cuentas merísticas seleccionadas por su alta estabilidad, en cuatro especies de Clupeiformes del litoral valdiviano

\begin{tabular}{|c|c|c|c|c|c|c|c|c|}
\hline \multirow{2}{*}{$\begin{array}{l}\text { Medidas y \% } \\
\text { Morfométricas }\end{array}$} & \multicolumn{2}{|c|}{ E. ringens } & \multicolumn{2}{|c|}{ E. maculatum } & \multicolumn{2}{|c|}{ S. sagax } & \multicolumn{2}{|c|}{ S. bentincki } \\
\hline & $\bar{X}$ & $\mathrm{CV}$ & $\bar{X}$ & $\mathrm{CV}$ & $\bar{X}$ & $\mathrm{CV}$ & $\bar{X}$ & $\mathrm{CV}$ \\
\hline En \% de LT: & & & & & & & & \\
\hline $\mathrm{LE}$ & 83,51 & 1,53 & 80,21 & 2,00 & 83,37 & 1,61 & 82,89 & 2,63 \\
\hline \multicolumn{9}{|l|}{ En \% de LE: } \\
\hline $\mathrm{DPD}$ & 52,25 & 3,25 & 45,66 & 4,31 & 49,68 & 6,30 & 56,60 & 4,33 \\
\hline DPA & 66,40 & 2,76 & 75,73 & 5,69 & 75,03 & 2,15 & 73,35 & 3,72 \\
\hline LC & 27,86 & 5,81 & 28,53 & 4,87 & 27,86 & 5,78 & 28,56 & 3,92 \\
\hline \multicolumn{9}{|l|}{ En \% de LC: } \\
\hline $\mathrm{DPO}$ & 16,78 & 6,44 & 21,95 & 6,33 & 28,32 & 8,16 & 23,80 & 5,97 \\
\hline $\mathrm{DPoO}$ & 61,75 & 2,90 & 56,68 & 8,84 & 47,93 & 5,42 & 5,08 & 4,97 \\
\hline Merísticas & Rango & $\mathrm{CV}$ & Rango & $\mathrm{CV}$ & Rango & $\mathrm{CV}$ & Rango & $\mathrm{CV}$ \\
\hline NRD & $16-18$ & $3,42 \%$ & $18-23$ & $5,05 \%$ & $16-20$ & $4,64 \%$ & $16-18$ & $3,40 \%$ \\
\hline NRV & 6-8 & $3,42 \%$ & 7 & $0,00 \%$ & $7-8$ & $2,77 \%$ & 8 & $0,00 \%$ \\
\hline NRA & $18-24$ & $6,53 \%$ & $15-19$ & $5,70 \%$ & $16-19$ & $3,94 \%$ & $16-19$ & $3,17 \%$ \\
\hline NRP & & & & & $15-20$ & $5,09 \%$ & $14-17$ & $3,76 \%$ \\
\hline NEV & & & $34-39$ & $3,02 \%$ & $30-34$ & $3,22 \%$ & & \\
\hline NEPD & & & $19-28$ & $9,64 \%$ & & & & \\
\hline
\end{tabular}

Measurements and meristic counts in four Clupeiform fishes from the Valdivian littoral, selected by its high stability 
En el texto se usan las siguientes abreviaturas: IZUA-PM, acrónimo de la Colección de Peces Marinos del Instituto de Zoología de la Universidad Austral de Chile (Leviton et al. 1985); CV, coeficiente de variación; DE, desviación estándar; PRM, probabilidad del rango medio; LT, longitud total; LE, longitud estándar; DPD, distancia predorsal; DPA, distancia preanal; LC, longitud de la cabeza; DPO, distancia preorbital; DPoO, distancia post-orbital; NBBI, número de branquispinas del brazo inferior del primer arco branquial.

\section{Resultados}

El resultado del análisis de los ejemplares de Clupeiformes depositados en la colección de peces indica que los 170 ejemplares estudiados, se agrupan en dos familias, con cuatro géneros y una especie en cada uno de ellos, así:

\section{Familia Engraulidae}

Engraulis ringens (Jenyns, 1842)

Familia Clupeidae

Ethmidium maculatum (Valenciennes, 1847)

Sardinops sagax (Jenyns, 1842)

Strangomera bentincki (Norman, 1936)

Como porcentaje en la longitud estándar, la distancia predorsal, la distancia preanal, la longitud de la cabeza, la distancia preorbital y la distancia postorbital, resultaron seleccionadas por su alta estabilidad y porque son comunes a todas las especies analizadas (Tabla 2).

Las características diagnósticas que permiten discriminar mejor mediante directa inspección visual a los distintos ejemplares representantes de las cuatro especies encontradas, dan lugar a la preparación de la siguiente clave:

\section{Clave ilustrada de reconocimiento de especies de Clupeiformes del litoral de Valdivia}

1a.- La región preorbital de la cabeza se proyecta hacia adelante, en un morro, producto que el mesetmoides se proyecta también bastante por delante del vómer (muy por delante del pre-maxilar), de modo que la boca queda en posición ventral, siendo una boca inferior. Longitud de la boca supera el 50\% de la longitud de la cabeza Fam. Engraulidae, Engraulis ringens

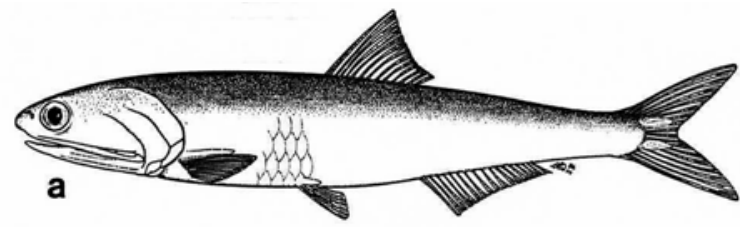

1b.- La región preorbital de la cabeza no se proyecta más delante de la mandíbula inferior, de modo que la abertura bucal queda en el extremo anterior del cuerpo, siendo una boca terminal. Longitud de la boca no supera el $50 \%$ de la longitud de la cabeza

Fam. Clupeidae 2

2a.- Con siete radios en la aleta pélvica y con notorios y tangibles escudetes predorsales en la línea media

Ethmidium maculatum

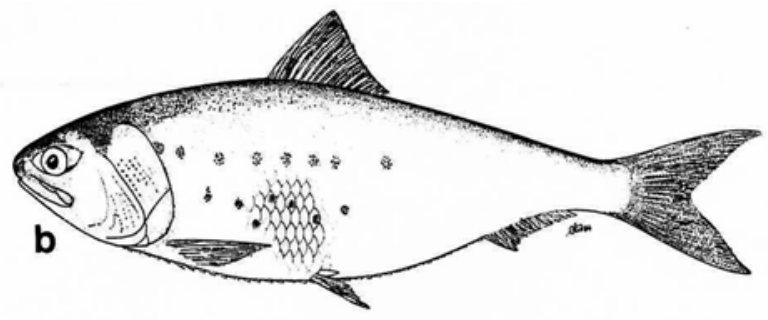

2b.- Con ocho radios pélvicos, rara vez siete; sin escudetes predorsales tangibles en la línea media 3

3a.- Con ocho radios pélvicos, rara vez siete; 18 a 20 radios pectorales; con estrías radiales en los opérculos Sardinops sagax

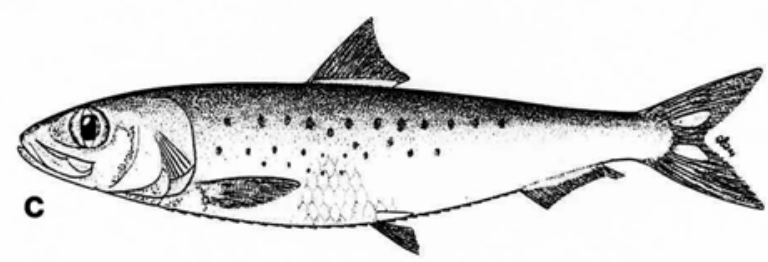

3b.- Ocho radios pélvicos; 14 a 17 radios pectorales; sin estrías radiales concéntricas en los opérculos

Strangomera bentincki

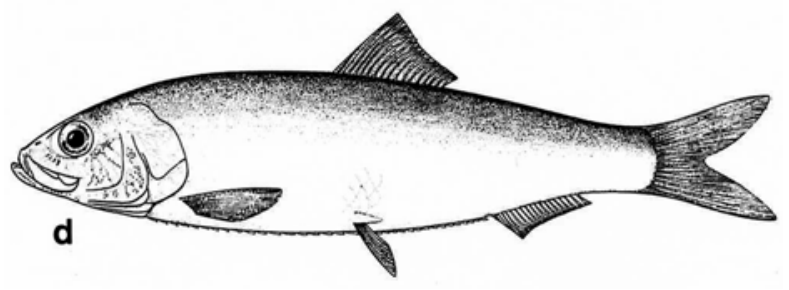


Aún con el uso de claves como la precedente, el reconocimiento de los especimenes suele presentar dificultades. Por eso, con el fin de proporcionar mayores herramientas complementarias morfológicas y merísticas, adjuntamos información especie por especie.

Las cuatro especies reconocibles a través de la clave precedente, tienen distribuciones geográficas hacia el norte de la costa de Valdivia. Aparentemente, solo Sardinops sagax tiene su límite sur en la zona valdiviana, sin que se haya precisado tal límite, justamente debido a los problemas de reconocimiento de las especies (Tabla 1). Lo señalado por Whitehead (1985) es algo contradictorio, pues mientras en el texto dice que se distribuye "southward to Mocha island, Chile, at $38^{\circ} 30^{\prime} \mathrm{S}$ “, en su correspondiente ilustración la muestra hasta poco más al sur de los $40^{\circ} \mathrm{S}$. Los ejemplares preservados en la colección de peces del Instituto de Zoología de la Universidad Austral de Chile constituyen documentación biológica para aumentar la distribución geográfica de la especie, hacia el sur, existiendo en la misma colección otros ejemplares que ratifican lo señalado en la Tabla 1.

\section{Comentarios}

Como se señaló anteriormente, hace 40 años no se sabía de la existencia de más de una o dos especies de peces del orden Clupeiformes en el litoral de Valdivia (Ethmidium maculatum y Strangomera bentincki). Hoy sabemos de la existencia de cuatro. Las cuatro especies corresponden a formas de distribución geográfica más septentrional que meridional, con respecto al litoral considerado. Al menos tres de ellas, Engraulis ringens, Ethmidium maculatum y Sardinops sagax, habitan también aguas del Perú, siendo la última habitante de aguas aún más al norte (Chirichigno \& Cornejo 2001). Solamente Strangomera bentincki, es de distribución más restringida a la costa central norte y central sur de Chile. En todo caso, S. sagax no ha sido encontrada al sur de lo que llamamos costa valdiviana. Por otro lado, especies de Clupeidae de hábitos más australes, como Sprattus fuegensis (Jenyns, 1842), parecen no invadir hacia el norte esta costa valdiviana, produciéndose una situación de alternancia, que en alguna medida coincide con la idea que dicha costa posee un sector de transición ictiofaunística (Pequeño 2001, 2004). Este tipo de transición litoral es más fácil de observar en formas de peces que viven bien apegadas a la costa, especialmente aquellas de hábitos bentónicos o muy dependientes de la orilla (por ej., peces de las familias
Cheilodactylidae, Chromidae, Dactyloscopidae, entre otras); pero hacia el mar abierto es menos frecuente de observar tal fenómeno. En este sentido, lo que ocurre con estos clupeiformes es importante, pues denotaría que la posible transición se extendería a especies que viven más allá del litoral mismo.

Las especies del orden Clupeiformes tienen importancia pesquera en todo el mundo (Whitehead 1985, Whitehead et al. 1988). Sin embargo, depende en alto grado de la cultura de los pueblos, la capacidad de las personas - pescadores - para reconocer las diferencias que indican que se tratan de especies diferentes. En el sur de Chile (Valdivia y al sur de dicha localidad), la pesca en mar abierto y sobre la plataforma continental, parece no haber existido de modo relevante antes de mediados del siglo XX (Mann 1954) y, por lo tanto, muchas de las especies pelágicas fueron generalmente desconocidas para la población. Mención aparte parece ser el tritre o machuelo, E. maculatum, especie que ha sido tradicionalmente capturada y sobre la cual existe conocimiento acerca de sus bondades cuando se le prepara ahumada. Dicha especie, según han observado directamente los autores, suele acercarse bastante a la costa e incluso entra en estuarios, como aquellos de los ríos Queule, Lingue, Valdivia y probablemente otros de más al sur (Pequeño \& Sáez 2004).

Sin embargo, al aparecer otras especies de Clupeiformes diferentes del tritre o machuelo, las capacidades para diferenciarlas son escasas y, hemos visto que hay pescadores que a las anchovetas les dicen sardinas, a los tritres jóvenes también les dicen sardinas y así, confunden entre estas especies. La situación de confusión en terreno es actual, de manera que no es trivial preocuparse de proveer herramientas para contribuir a la necesaria diferenciación, especialmente cuando se trata de llevar registros con fines estadísticopesqueros. En esta oportunidad, a diferencia de todas las anteriores, se reúnen todas las especies del litoral valdiviano, que ha sido especialmente activo en extracciones en los últimos dos decenios, en una clave de reconocimiento que considera aspectos de fácil tratamiento y además, cuenta con un agregado a modo de auxiliar que complemente a la clave, basado en criterios cuantitativos simples, pero con capacidad de discriminación que conlleva confiabilidad. La nueva clave que aquí se presenta, se diferencia de las anteriores por el mayor número muestreal utilizado en la obtención de características morfológicas y merísticas, por la forma en que se seleccionaron 
aquellas características y por el hecho que tales características no habían sido utilizadas por otros autores.

El hecho que en tres pasos de fácil acceso, considerados por la clave, sea posible separar a dos familias hasta el nivel de especie, permite suponer que ésta será de utilización práctica, tanto en el laboratorio como en terreno, cuestión que podrá ser probada solamente con el paso del tiempo y la difusión correspondiente.

\section{Agradecimientos}

Agradecemos el permiso de la Organización de las Naciones Unidas para la Agricultura y la Alimentación (FAO), a través de su Departamento de Pesquerías, por otorgar autorización para reproducir las figuras de los peces; a Michel Lamboeuf (FAO), por su gestión de apoyo a esta publicación; la colaboración de León Matamala (Instituto de Zoología, UACH) y Patricia Pacheco (P. Univ. Católica de Valparaíso). Dos revisores anónimos comprometen la gratitud de los autores, debido a sus valiosas opiniones. Estos son resultados parciales del Proyecto DID-S-200223 de la Universidad Austral de Chile, otorgado al co-autor.

\section{Literatura citada}

Boré D \& C Martínez. 1980. Catálogo de recursos pesqueros de Chile, 40 pp. CORFO-IFOP, Santiago.

Chirichigno N \& M Cornejo. 2001. Catálogo comentado de los peces marinos del Perú. Publicación Especial. Instituto del Mar del Perú: 1-314.

Chirichigno N \& J Vélez. 1998. Clave para identificar los peces marinos del Perú. Publicación Especial. Instituto del Mar del Perú: 1-502.

De Buen F. 1958. Peces de la superfamilia Clupeoidae en aguas de Chile. Revista de Biología Marina 8 (1-3): 83110.

Figueroa V. 1985. Papel de probabilidad normal, su uso. Comunicaciones Instituto de Estadística, Facultad de Ciencias Económicas y Administrativas, Universidad Austral de Chile, Serie Informes Técnicos: 1-20.

Harding JP. 1949. The use of probability paper for the graphical analysis of polymodal frequency distributions. Journal of the Marine Biological Association of the United Kingdom 28: 141-153.
Leible M \& E Alveal. 1982. Catálogo de los peces que habitan las aguas costeras y dulceacuícolas en la Provincia de Concepción, 104 pp. Universidad Católica, Departamento de Biología y Tecnología del Mar, Talcahuano.

Leviton AE, RH Gibbs Jr, E Heald \& CE Dawson. 1985. Standards in herpetology and ichthyology: Part I, Standard symbolic codes for institution resource collections in herpetology and ichthyology. Copeia 1985 (3): 802-832.

López RB 1963. Problemas de la distribución geográfica de los peces marinos suramericanos. Boletín Instituto de Biología Marina Mar del Plata 7: 57-62.

Mann G. 1954. La vida de los peces en aguas chilenas, 342 pp. Instituto de Investigaciones Veterinarias y Universidad de Chile, Santiago.

Pequeño G. 2001. El "bilagai” Cheilodactylus variegatus Valenciennes, 1833, en la costa de Valdivia (Osteichthyes: Cheilodactylidae). Estudios Oceanológicos 20: 23-28.

Pequeño G. 2004. El estatus taxonómico de Graus fernandezianus Philippi, 1887; nuevo registro geográfico y comentarios sobre Graus nigra Philippi, 1887 (Osteichthyes: Perciformes), en Chile. Gayana 68(1): 6369.

Pequeño G \& S Sáez. 2004. Peces marinos comunes del litoral de Valdivia, 87 pp. Ediciones Surambiente, Corporación Ambiental del Sur, Santiago.

Sáez P. 1988. Somatometría y morfología de la sardina española Sardinops sagax (Jenyns, 1842) de la zona de Iquique, Chile (Clupeidae, Clupeiformes). Revista de Biología Marina 24(1): 73-90.

Simpson JG, M Carreño, HS Schlotfeldt \& E Gil. 1967. Distribución geográfica mensual y trimestral y composición de las frecuencias de longitudes por mes de las capturas de anchoveta por zona desembarcada en Arica, de Agosto 1963 a Diciembre 1965. Publicación Instituto de Fomento Pesquero 25: 1-91.

Sokal R \& F Rohlf. 1979. Biometría: principios y métodos estadísticos en la investigación biológica, 832 pp. H. Blume Ediciones, Madrid.

Whitehead JPJ. 1985. Clupeoid fishes of the world. An annotated and illustrated catalogue of herrings, sardines, pilchards, sprats, anchovies and wolf-herrings. Part 1 Chirocentridae, Clupeidae and Pristigasteridae. FAO Fisheries Synopsis 125, 7(1): 1-303. 
Whitehead JPJ. 1964. A new genus and subgenus of clupeid fishes and notes on the genera Clupea, Sprattus and Clupeonella. Bulletin of the British Museum (Natural History) 13 (7): 321-330.

Whitehead JPJ, J Nelson \& $T$ Wongratana. 1988. Clupeoid fishes of the world (Suborder Clupeoidei). An annotated and illustrated catalogue of the herrings, sardines, pilchards, sprats, anchovies and wolf-herrings.
Part 2, Engraulididae. FAO Fisheries Synopsis 125, 7(2): 305-579.

Yañez E, M García \& M Barbieri. 1997. Fluctuaciones de pesquerías pelágicas y del medioambiente en el Pacífico suroriental. En: Tarifeño E (ed). Gestión de sistemas oceanográficos del Pacífico oriental, pp. 223-236. Comisión Oceanográfica Intergubernamental (IOC) y Universidad de Concepción, Concepción.

Recibido el 28 de noviembre de 2006 y aceptado el 25 de septiembre de 2007 